\title{
Prostacyclin infusion in patients with acute myocardial infarction
}

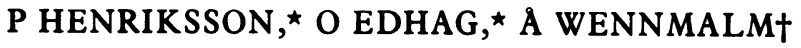 \\ From the Departments of ${ }^{\star}$ Medicine and + Clinical Physiology, Karolinska Institute, Huddinge University Hospital, \\ Huddinge, Sweden
}

SUMMARY To investigate whether prostacyclin protects ischaemic myocardium in humans the effect of prostacyclin or placebo was studied in two groups of patients with acute myocardial infarction who presented within six and 16 hours of the onset of symptoms. Intravenous infusion of prostacyclin or placebo was started immediately after admission at a rate corresponding to $4-5 \mathrm{ng} / \mathrm{kg} / \mathrm{minute}$. The infusion was maintained for 72 hours. Clinical status, electrocardiograms, plasma enzyme activity, infarct extension during the infusion, and reinfarction after the infusion were studied. Prostacyclin was well tolerated by most patients: neither systemic blood pressure nor heart rate differed between the two groups. In the 11 patients who received treatment within six hours of the onset of symptoms prostacyclin significantly lowered the maximum plasma activities of creatine kinase $M B$ and lactate dehydrogenase. In the 19 patients who received treatment 6-16 hours after the onset of symptoms prostacyclin had no such effect. None of the patients receiving prostacyclin had an extension of the infarction during the infusion, whereas four patients receiving placebo did; this difference was significant.

These data are the first to provide evidence that prostacyclin might limit myocardial injury in patients with acute myocardial infarction.

Prostacyclin is a metabolite of arachidonic acid that is formed in vascular endothelium throughout the body. ${ }^{1-3}$ Prostacyclin has vasodilative and platelet antiaggregatory properties, and in view of these actions the compound may prevent platelet aggregation and thrombosis in healthy vessels. Vascular formation of prostacyclin is reduced in atherosclerosis, ${ }^{4-6}$ and this has been proposed as a factor responsible for the increased incidence of thrombosis in this state.

The actions mentioned above have also prompted the proposal of prostacyclin as a suitable agent for the treatment of acute myocardial infarction. The findings concerning endothelial production of prostacyclin in healthy and atherosclerotic vessels suggested that such treatment might restore lost prostacyclin activity in the cardiovascular system, especially in the coronary circulation. In fact, prostacyclin

Requests for reprints to Dr P Henriksson, Department of Medicine, Karolinska Institute, Huddinge University Hospital, S-141 86 Huddinge, Sweden.

Accepted for publication 9 October 1984 reduces the size of experimental infarction in various animal models. ${ }^{7-10}$ On the other hand, the administration of prostacyclin to humans with various signs of ischaemic heart disease has yielded inconclusive results. ${ }^{11-13}$

To evaluate the effect of prostacyclin in patients with acute myocardial infarction, we performed a double blind investigation in $\mathbf{3 0}$ patients.

\section{Patients and methods}

The study was performed during 1983-84 at this hospital. Patients admitted to the hospital coronary care unit and meeting the selection criteria of the study were invited to participate. Informed consent was obtained from all patients. The study protocol was approved by the local human investigations committee.

SELECTION OF PATIENTS

Patients admitted with chest pain typical of acute infarction ( $>30 \mathrm{~min}$ duration) and with confirmative electrocardiogram or plasma enzyme activity or both were included provided that treatment could be 
started within 16 hours of the onset of symptoms. The age limits were 18-75 years; female patients were included only if postmenopausal. Either $(a)$ new or presumably new $\mathrm{Q}$ waves of at least $0.04 \mathrm{~s}$ duration and amplitude amounting to $>25 \%$ of the following $R$ wave in either (i) leads II, III, and aVF, (ii) leads I and aVL, or (iii) two or more precordial leads, or $(b)$ ST segment elevation of $>2 \mathrm{mV}$ in two leads were the confirmative electrocardiographic criteria used. A rise in plasma activity to at least twice the upper normal limit of the hospital laboratory's reference value for two of the enzymes-creatine kinase, aspartate aminotransferase, and lactate dehydrogenase-or appreciably increased myocardial specific creatine kinase MB activity was accepted as evidence of acute myocardial infarction.

Patients with an earlier acute myocardial infarction within the past two months, as well as those with psychiatric, endocrinological or infectious or malignant diseases, were excluded.

\section{RANDOMISATION AND STRATIFICATION}

A randomisation protocol was prepared before the study and was kept by the pharmacist delivering the drug to the coronary care unit during the entire investigation. The randomisation protocol was adapted to yield 15 patients in each of the prostacyclin and placebo groups. The investigation protocol also prescribed a separate study to compare the results of patients receiving prostacyclin with those receiving placebo within six hours of the onset of symptoms. These patients were, however, not randomised separately.

\section{ADMINISTRATION OF PROSTACYCLIN OR}

PLACEBO

Prostacyclin (synthetised by the Upjohn Company and formulated by the Wellcome Foundation), stored as a freeze-dried powder, was dissolved in glycine buffer $\mathrm{pH} 10.5$ immediately before infusion. A fresh solution was prepared every 12 hours during the infusion period. Glycine buffer at $\mathrm{pH} 10.5$ was infused as the placebo. The infusion was given into a peripheral arm vein using a volume controlled pump (IMED 922) at a rate corresponding to $1 \mathrm{ng} / \mathrm{kg} / \mathrm{min}$ and increased stepwise over one hour to a maximum of $5 \mathrm{ng} / \mathrm{kg} / \mathrm{min}$. If systolic blood pressure fell or heart rate increased by $>10 \%$ before the maximum infusion rate had been reached the infusion was maintained at the current rate. The overall infusion time was $\mathbf{7 2}$ hours. During the last four hours the infusion rate was gradually tapered off in order to avoid rebound phenomena. In addition to the infusion of prostacyclin, the patients received routine clinical and pharmacological treatment for acute myocardial infarction and were man- aged by the ordinary staff of the coronary care unit. The investigators did not influence the routine care.

\section{EVALUATION OF CLINICAL STATUS AND}

\section{LABORATORY FINDINGS}

All patients underwent a baseline clinical and laboratory evaluation before infusion of the drug was started comprising a physical examination, a standard 12 lead electrocardiogram, chest $x$ ray examination, routine chemical blood examination, and measurement of plasma activity of creatine kinase $\mathrm{MB}$, creatine kinase, aspartate aminotransferase, and lactate dehydrogenase. During the infusion of prostacyclin a 12 lead electrocardiogram was recorded daily. Plasma creatine kinase $M B$ activity was measured in samples taken 8, 16, and 24 hours after the start of the infusion. Plasma creatine kinase, aspartate aminotransferase, and lactate dehydrogenase activities were measured daily until discharge. At the end of the study (30 days after admission) a physical examination, including evaluation of the patient's functional status, and an electrocardiogram were performed. The 12 lead electrocardiograms were analysed using a QRS scoring system ${ }^{14}$ in which each recording was scored from 0 to 29 , with a value $>2$ strongly suggesting myocardial infarction. QRS scoring was performed on electrocardiograms recorded on days 3,7 , and 30 after the start of the infusion. ST segment elevation was measured as the sum of the vertical distances between the isoelectric line and the midpoint of the ST segment in the two leads showing the most prominent elevations on day 1. ST elevation was assessed on days 1,2 , and 3 after the start of the infusion. All electrocardiographic evaluations were performed by a trained interpreter who was not a member of the investigating team and was unaware of the type of treatment the patients had received.

\section{Results}

\section{COMPARABILITY OF THE GROUPS}

Table 1 shows the result of the randomisation procedure. The number of patients, their sex distribution and age, as well as the time from the onset of symptoms to the start of infusion did not differ between the prostacyclin and placebo groups. The number of patients receiving infusion within six hours of the onset of symptoms was slightly greater in the prostacyclin group. Some patient characteristics related to an increased incidence of ischaemic heart disease were over-represented in the prostacyclin group, but the difference is not significant. Table 2 shows the number of patients receiving beta blocking drugs before or during the infusion of prostacyclin or placebo. The proportion of patients given beta blocking drugs did not differ between the two groups. The 
Table 1 Group characteristics in patients with acute myocardial infarction randomised to receive prostacyclin or placebo and the occurrence of factors related to increased incidence of myocardial infarction in the two groups. Figures are numbers of patients

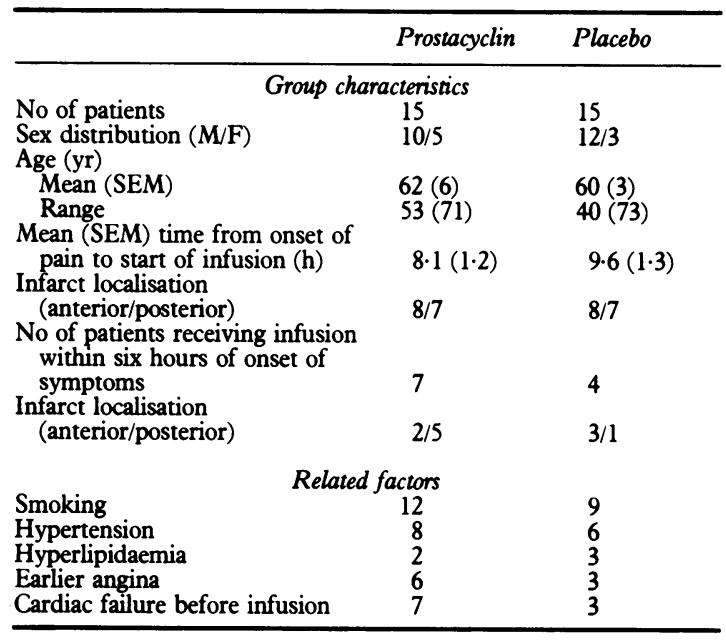

proportion of patients receiving beta blocking drugs was in fact slightly higher in the placebo group, and this tendency was reinforced during days 2 and 3 of infusion. The difference did not, however, reach statistical significance.

\section{DRUG.TOLERANCE}

Of the patients in the prostacyclin group, 12 could be given the drug at a rate of $5 \mathrm{ng} / \mathrm{kg} /$ minute without exceeding the predetermined systolic blood pressure value or pulse rate. In one patient the infusion rate was never increased above $4 \mathrm{ng} / \mathrm{kg} /$ minute because of a tendency to hypotension, and in two other patients the rate had to be reduced to $4 \mathrm{ng} / \mathrm{kg} /$ minute for the same reason. Figure 1 shows the values for the systolic and diastolic blood pressure and heart rate. There were no differences in these variables before, during, or after the infusion. Table 3 shows the cardiac stress index (the product of the systolic blood pressure and the heart rate) during the infusion in the two groups. Again there was no difference between the groups.

\section{CLINICAL OBSERVATIONS}

Table 4 shows the occurrence of congestive heart failure before and after the study. Two patients were receiving treatment for heart failure when they entered the study; both were in the prostacyclin group. Another five patients in this group had clinical or radiographic findings or both indicating heart failure on entering the study. The number of patients in the prostacyclin group requiring treatment for heart failure did not increase during the course of the infusion. In the placebo group three patients had clinical or radiographic evidence of heart failure on entering the study. During the course of the infusion, this number increased to eight (Table 4).

Table 2 Administration of beta blocking drugs in the groups receiving prostacyclin or placebo. Figures are numbers of patients (mean 24 hour dosages in $\mathrm{mg}$ given in parentheses)

\begin{tabular}{|c|c|c|c|c|c|}
\hline Drug & Time $(h)^{\star}$ & Preinfarction & Day 1 & Day 2 & Day 3 \\
\hline \multicolumn{6}{|c|}{ Prostacyclin group } \\
\hline Metoprolol & $0-16$ & $1(200)$ & $1(200)$ & $4(125)$ & $4(125)$ \\
\hline & $0-6$ & $1(200)$ & $1(200)$ & $2(175)$ & $2(175)$ \\
\hline Pindolol & $0-16$ & $1(10)$ & $1(10)^{\prime}$ & $1(10)$ & $1(10)$ \\
\hline Atenolol & $0-16$ & $1(100)$ & $1(100)$ & $1(10)$ & $1(10)$ \\
\hline & $0-6$ & 0 & 0 & & \\
\hline None & $\begin{array}{l}0-16 \\
0-6\end{array}$ & $\begin{array}{r}12 \\
6\end{array}$ & $\begin{array}{r}12 \\
6\end{array}$ & $\begin{array}{l}9 \\
5\end{array}$ & $\begin{array}{l}9 \\
5\end{array}$ \\
\hline \multicolumn{6}{|c|}{ Placebo group } \\
\hline Metoprolol & $\begin{array}{l}0-16 \\
0-6\end{array}$ & $\begin{array}{l}1(100) \\
0\end{array}$ & $2(125)$ & $\begin{array}{l}7(107) \\
4(75)\end{array}$ & $\begin{array}{l}7(121) \\
3(83)\end{array}$ \\
\hline Alprenolol & $\begin{array}{l}0-16 \\
0-6\end{array}$ & $2_{0}^{2}(400)$ & $2(400)$ & $2(400)$ & $2(400)$ \\
\hline Timolol & $0-16$ & 0 & 0 & 0 & $1(20)$ \\
\hline Practololt & $0_{0-16}^{0-6}$ & 0 & 0 & 0 & 0 \\
\hline Practolort & $\begin{array}{l}0-16 \\
0-6\end{array}$ & $\begin{array}{l}0 \\
0\end{array}$ & $\begin{array}{l}0 \\
1(15)\end{array}$ & $\begin{array}{l}0 \\
0\end{array}$ & $\begin{array}{l}0 \\
0\end{array}$ \\
\hline None & $0-16$ & 12 & 10 & 5 & 4 \\
\hline & $0-6$ & 4 & 3 & 0 & 1 \\
\hline
\end{tabular}

$\star$ From onset of chest pain to start of infusion.

tGiven intravenously. 

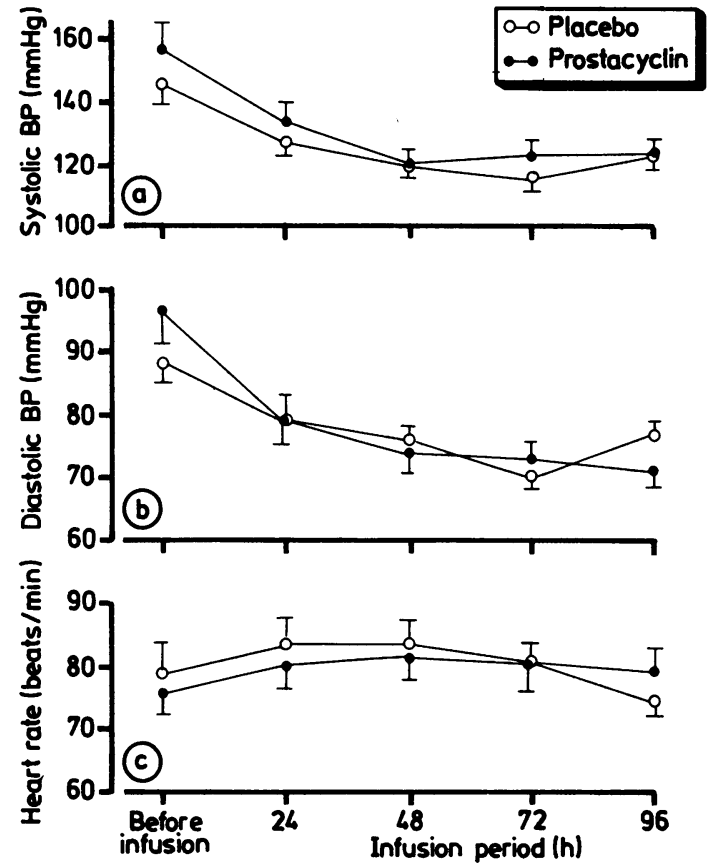

Fig. 1 (a) Systolic and (b) diastolic blood pressure (BP) and (c) heart rate from 0-96 hours after the onset of intravenous infusion of prostacyclin (4-5 $\mathrm{ng} / \mathrm{kg} / \mathrm{min}$ ) or placebo during 72 hours in patients with acute myocardial infarction.

Table 3 Cardiac stress index (expressed as the product of systolic blood pressure ( $\mathrm{mm} \mathrm{Hg}$ ) and heart rate (beats/min)) before (day 0), during (day 1-3), and after (day 4) infusion of prostacyclin (4-5 ng/kg/min) or placebo in patients with acute myocardial infarction. Values are mean (SEM)

\begin{tabular}{lll}
\hline & Prostacyclin & Placebo \\
\hline Day 0 & $11677(1248)$ & $11756(1268)$ \\
Day 1 & $10313(896)$ & $11647(928)$ \\
Day 2 & $10572(770)$ & $10437(787)$ \\
Day 3 & $9857(519)$ & $9172(711)$ \\
Day 4 & $9307(420)$ & $9828(585)$ \\
\hline
\end{tabular}

Table 4 Occurrence of congestive heart failure (CHF) among patients treated for acute myocardial infarction with prostacyclin or placebo. Figures are numbers of patients

\begin{tabular}{lll}
\hline & Prostacyclin & Placebo \\
\hline Treatment of CHF: & & \\
Before study & 2 & 0 \\
After infusion & 6 & 8 \\
Signs of CHF: & 7 & 3 \\
Before study & 2 & 5 \\
After infusion & & \\
\hline
\end{tabular}

^Additional patients.
Table 5 Occurrence of infarct extension, reinfarction, and deaths in patients with acute myocardial infarction treated with prostacyclin or placebo. Figures are numbers of patients

\begin{tabular}{lll}
\hline & Prostacyclin & Placebo \\
\hline Extension of infarction (during & & \\
the infusion) & $0^{\star}$ & 4 \\
Reinfarction (after the infusion) & 2 & 0 \\
Deaths & 0 & 1 \\
\hline
\end{tabular}

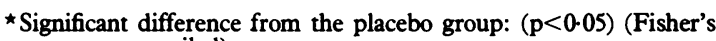
exact test, one tailed).

EXTENSION OF INFARCTION AND REINFARCTION Extension of the acute myocardial infarction, as evidenced by a new episode of typical chest pain in conjunction with a new rise in plasma creatine kinase MB or creatine kinase activity, occurred during the infusion in three patients receiving placebo. In addition, another patient receiving placebo, having improved clinically during the initial 24 hours of infusion, developed persistent pain for more than 12 hours and a fatal ventricular rupture on day 2; this case was also regarded as an extension of the infarct. None of the patients receiving prostacyclin developed similar signs of infarct extension during the infusion (Table 5). This difference in infarct extension (4 vs 0 ) is significant $(p<0.05$, Fisher's exact test, one tailed). From the end of the infusion until day 30 , none of the
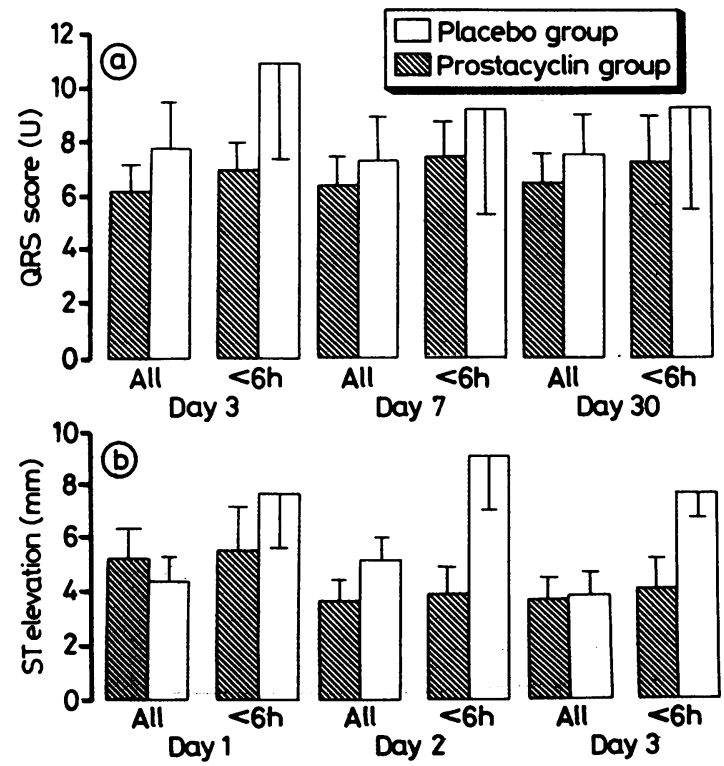

Fig. 2 (a) QRS score and (b) sum of ST elevations in all patients with acute myocardial infarction infused with prostacyclin (4-5 ng/kg/min) or placebo during 72 hours and in patients receiving infusion within six hours $(<6 h)$ after the onset: of symptoms. Values are mean (SEM). 

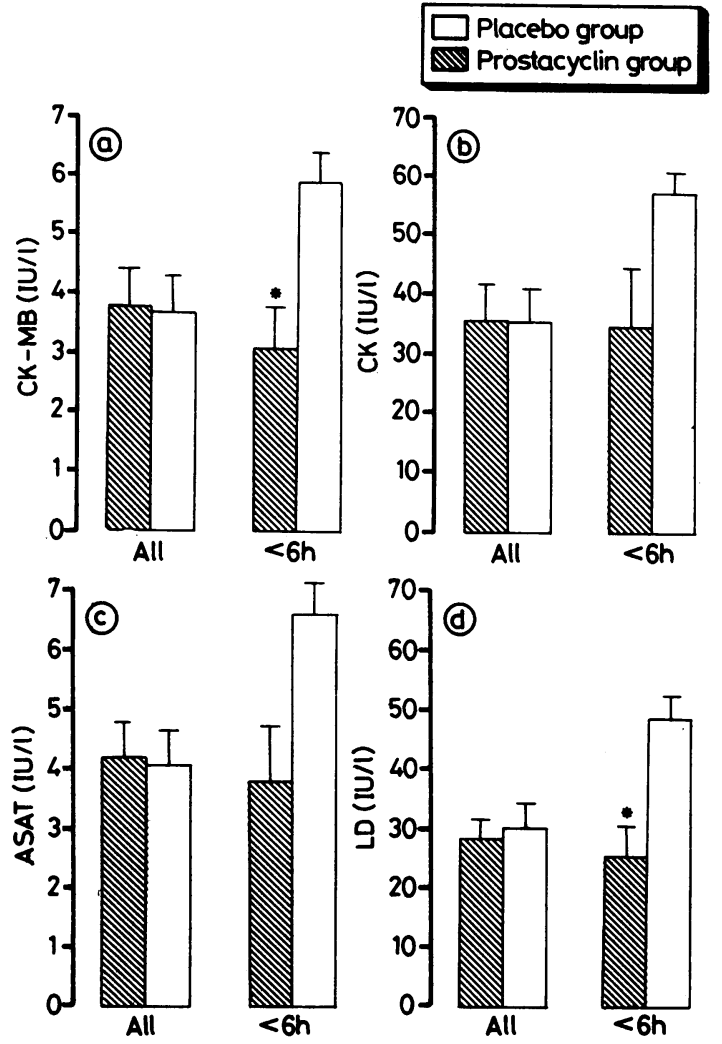

Fig. 3 Peak plasma activities of (a) myocardial specific creatine kinase $(C K-M B),(b)$ total creatine kinase $(C K)$, (c) aspartate aminotransferase (ASAT), and (d) lactate dehydrogenase $(L D)$ in all patients with acute myocardial infarction treated with prostacyclin or placebo and in patients receiving infusion within six hours $(<6 h)$ after the onset of symptoms. ${ }^{\star} p<0.05$, significant difference from placebo group. patients in the placebo group developed reinfarction. In the prostacyclin group, two patients developed a reinfarction in the same area as that injured initially (on day 6 and 21).

\section{LABORATORY FINDINGS}

Figure 2 shows the QRS score and sum of ST elevations in the two groups. The QRS score was slightly but not significantly lower on all occasions in the patients receiving prostacyclin, both in the groups as a whole and in those receiving an infusion within six hours of the onset of symptoms. The sum of ST eleva tions did not show any uniform pattern in the group. as a whole but tended to be lower in the prostacyclin group in patients receiving an infusion within six hours of onset of symptoms.

The maximum plasma activity of creatine kinase $M B$, creatine kinase, aspartate aminotransferase, and lactate dehydrogenase did not differ between the groups as a whole (Fig. 3). Nevertheless, in the patients who received an infusion within six hours of the onset of pain both plasma creatine kinase $\mathrm{MB}$ and lactate dehydrogenase activity was significantly ( $p<0.05$ and 0.05 respectively) lower (Fig. 3).

The time course of the release of enzymes in the patients receiving an infusion within six hours of the onset of pain was also determined (Fig. 4). During the first 24 hours of infusion the increase in creatine kinase $\mathrm{MB}$ activity was smaller in the prostacyclin group than in the placebo group, as was the increase in plasma lactate dehydrogenase activity (Fig. 4). The initial mean value did not differ between the groups, but the peak value was lower in the prostacyclin group. Interestingly, after the end of the infusion lactate dehydrogenase activity reached almost identical values in the prostacyclin and the placebo groups.
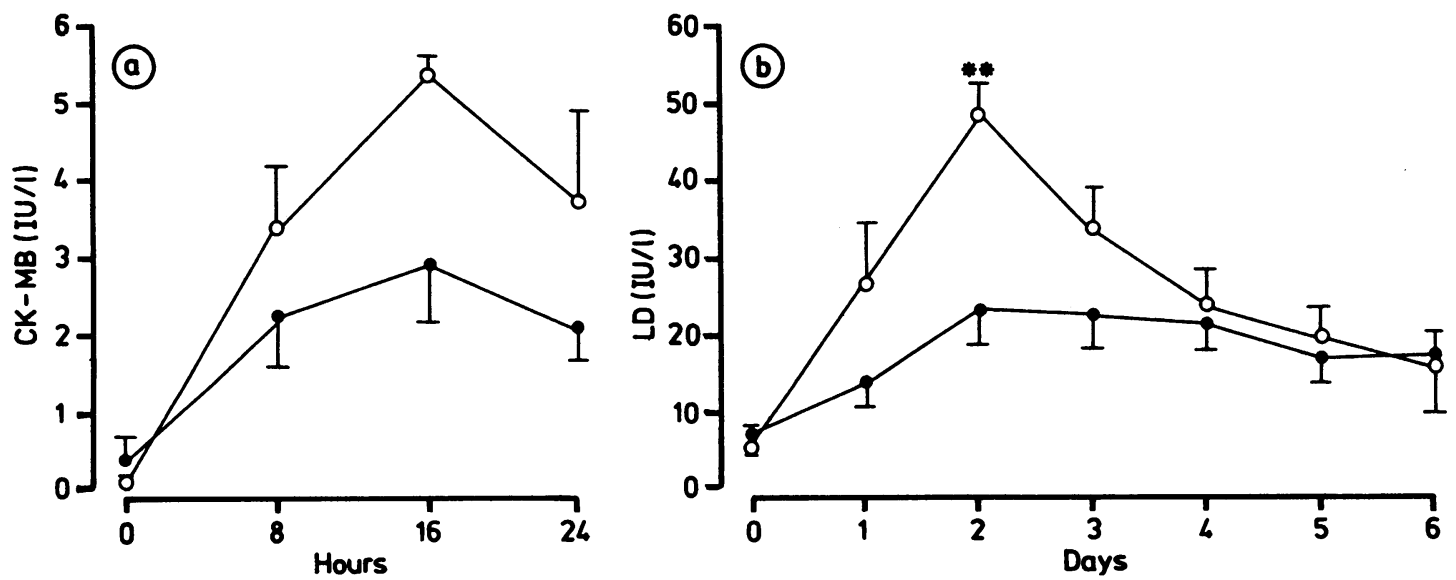

Fig. 4 Plasma ensyme activity in relation to time in patients with acute myocardial infarction given prostacyclin $(O)$ or placebo $(O)$ within six hours after the onset of symptoms: (a) plasma activity of myocardial specific creatine kinase (CK-MB) immediately before (O hours) and during the first 24 hours of infusion of prostacyclin or placebo and (b) plasma activity of lactate dehydrogenase (LD) in the same patients in the basal state $($ day 0$)$ and during the first six days after the onset of symptoms. $\star \star p<0.01$, significant difference from prostacyclin group. 


\section{Discussion}

Patients receiving prostacyclin within six hours of the onset of symptoms had significantly lower maximum plasma activity of creatine kinase MB and lactate dehydrogenase than the placebo group. We regard these lower values for plasma creatine kinase $M B$ and lactate dehydrogenase activity in the prostacyclin group as indicating smaller infarcts among these patients compared with the placebo group. As evident from Fig. 3 the patients receiving early placebo infusion had higher maximum plasma creatine kinase $\mathrm{MB}$, creatine kinase, aspartate aminotransferase, and lactate dehydrogenase activities than the group as a whole. This is probably because patients with larger infarctions-and hence higher maximum plasma enzyme values-experience more pronounced symptoms and therefore present earlier. The proportion of patients with anterior wall infarctions was greater in the placebo group; whether or not this may have influenced the data cannot be judged at present. The proportion of patients taking beta blocking drugs was not higher in the prostacyclin group. Thus the observed difference in plasma enzyme activity between the groups can hardly be explained by a more active beta blocking regimen in the prostacyclin group. A direct cardioprotective action of prostacyclin in acute myocardial infarction therefore seems possible.

In addition to the differences in maximum plasma creatine kinase $M B$ and lactate dehydrogenase activities, the time courses of the plasma activity of these enzymes are notable. Thus the curve for creatine kinase $\mathrm{MB}$ activity in the prostacyclin group was flatter than in the placebo group. In addition, the peak of the plasma lactate dehydrogenase curve in patients infused with prostacyclin within six hours was lower than in those given placebo. Interestingly, the plasma lactate dehydrogenase values in the prostacyclin group were numerically lower during the entire infusion, approaching those in the placebo group after the infusion.

Another important finding was that patients receiving prostacyclin appeared to be protected against infarct extension during the infusion. This was evidenced by a significantly lower incidence of infarct extension in patients receiving prostacyclin than in those receiving placebo. The lower incidence of infarct extension seems to accord well with the observed lower maximum plasma creatine kinase MB and lactate dehydrogenase activities discussed above, in as much as they indicate that myocardium at risk at the onset of the infusion is protected by the active drug and, consequently, that enzyme release and a possible tendency to infarct extension are depressed. If this hypothesis is correct patients receiving pros- tacyclin should run an increased risk of reinfarction after the infusion. The incidence of reinfarction tended to increase in the present group of patients receiving prostacyclin; two developed reinfarction after the infusion compared with none of the patients who had received placebo; the difference was, however, not significant.

A drug with vasodilative activity, like prostacyclin, can be expected to induce a decrease in peripheral vascular resistance and a reflex increase in cardiac output. The latter is certainly not desirable in acute myocardial infarction since the increase in cardiac output may require more dynamic work by the heart. In the present study prostacyclin did not affect blood pressure and heart rate more than placebo-that is, with the infusion rate limits applied in the present study the drug probably does not interfere appreciably with the central haemodynamics. Bradycardia was not a general finding in the placebo group, probably because the patients were not routinely given beta blocking drugs during the acute phase. Furthermore, prostacyclin did not induce cardiac failure or even aggravate signs of failure. In fact all patients in the placebo group with signs of cardiac failure before infusion also required treatment for this afterwards; in addition, five more patients in this group developed evidence of cardiac failure during the infusion. In contrast, of the seven patients in the prostacyclin group who had signs of cardiac failure before the infusion, three did not require treatment afterwards.

Although the present study points to a beneficial effect of prostacyclin in patients with acute myocardial infarction, the possible underlying mechanism is unknown. Since a myocardial saving effect of prostacyclin is indicated in patients receiving treatment within six hours of the onset of symptoms some conceptually related postinfusion treatment (that is, treatment aimed to counteract platelet aggregation and formation of thromboxane A2) should be given that can keep salvaged myocardium in a metabolic condition that allows contractile work to be maintained. In men with unstable angina aspirin has been shown to protect against acute myocardial infarction and death ${ }^{15}$; this drug should be effective also in the present situation, in which myocardium at risk may persist after the infusion of prostacyclin.

OE was supported by the Swedish National Association against Heart and Chest Diseases, and $A W$ by the Swedish Medical Research Council. Prostacyclin was kindly provided by the Upjohn Company.

\section{References}

1 Moncada S, Gryglewski R, Bunting S, Vane JR. An enzyme isolated from arteries transforms prostaglandin endoperoxides to an unstable substance that inhibits 
platelet aggregation. Nature 1976; 263: 663-5.

2 Gryglewski RJ, Bunting S, Moncada S, Flower RJ, Vane JR. Artcrial walls are protected against deposition of platelet thrombi by a substance (prostaglandin $\mathrm{X}$ ) which they make from prostaglandin endoperoxides. Prostaglandins 1976; 12: 685-713.

3 Moncada S, Higgs EA, Vane JR. Human arterial and venous tissues generate prostacyclin (prostaglandin X) a potent inhibitor of platelet aggregation. Lancet 1977; i: 18-21.

4 Gryglewski RJ, Dembińska-Kièć A, Zmuda A, Gryglewska T. Prostacyclin and thromboxane $A_{2}$ biosynthesis capacities of heart, arteries and platelets at various stages of experimental atherosclerosis in rabbits. Atherosclerosis 1978; 31: 385-94.

5 Sinzinger H, Feigl $W$, Silberbauer K, Oppolzer R, Winter M, Auerswald W. Prostacyclin ( $\mathrm{PGI}_{2}$ )-generation by different types of human atherosclerotic lesions. Exp Pathol 1980; 18: 175-80.

6 Larrue J, Rigaud M, Daret D, Demond J, Durand J, Bricaud $H$. Prostacyclin production by cultured smooth muscle cells from atherosclerotic rabbit aorta. Nature 1980; 285: 480-2.

7 Ogletree ML, Lefer AM. Prostaglandin-induced preservation of the ischemic myocardium. Circ Res 1978; 42: 218-24.

8 Ogletree ML, Lefer AM, Smith JB, Nicolaou KC.
Studies on the protective effect of prostacyclin in acute myocardial ischemia. Eur $\mathcal{F}$ Pharmacol 1979; 56: 95-103.

9 Araki H, Lefer AM. Role of prostacyclin in the preservation of ischemic myocardial tissue in the perfused cat heart. Circ Res 1980; 47: 757-63.

10 Jugdutt BI, Hutchins GM, Bulkley BH, Becker LC. Dissimilar effects of prostacyclin, prostaglandin $E_{1}$ and prostaglandin $E_{2}$ on myocardial infarct size after coronary occlusion in conscious dogs. Circ Res 1981; 49: 685-700.

11 Szczeklik A, Szczeklik J, Nizankowski R, Gluszko P. Prostacyclin for acute coronary insufficiency. Artery 1980; 8: 7-11.

12 Szczeklik A, Szczeklik J, Nizankowski R. Prostacyclin, nitroglycerin and effort angina [Letter]. Lancet 1981; i: 1006.

13 Chierchia S, Patrono C, Crea F, et al. Effects of intravenous prostacyclin in variant angina. Circulation 1982; 65: 470-7.

14 Wagner GS, Freye CJ, Palmeri ST, et al. Evaluation of a QRS scoring system for estimating myocardial infarct size. I. Specificity and observer agreement. Circulation 1982; 65: 342-7.

15 Lewis HD Jr, Davis JW, Archibald DG, et al. Protective effects of aspirin against acute myocardial infarction and death in men with unstable angina. $N$ Engl f Med 1983; 309: 396-403. 